\author{
Article \\ Doi 10.5943/sif/1/1/16 \\ Copyright $($ Mushroom Research Foundation 2016
}

\title{
Production of Catecholate Siderophores by a Manglicolous fungus Emericella nidulans: A Novel Observation
}

\author{
Trivedi $\mathrm{HB}^{1}$, Vala $\mathrm{AK}^{1}$ and Dave $\mathrm{BP}^{1 *}$ \\ ${ }^{I}$ Department of Life Sciences, Maharaja Krishnakumarsinhji Bhavnagar University, Bhavnagar-364 002, India
}

Trivedi HB, Vala AK, Dave BP 2016 - Production of Catecholate Siderophores by a Manglicolous fungus Emericella nidulans: A Novel Observation. Studies in Fungi 1(3), 146-149, Doi $10.5943 / \mathrm{sif} / 1 / 1 / 16$

\begin{abstract}
Mangrove ecosystem is a rich source of manglicolous fungi. While much information is available on diversity of these fungi, their siderophores have not been explored yet. The present study was carried out with a view to explore siderophore production potential of manglicolous fungi along Ghogha Coast, Gulf of Khambhat, Gujarat, India. From four different mangrove samples viz. rhizosphere, rhizoplane, water and sediment, thirteen fungi belonging to Ascomycota were isolated, which were all siderophore producers. Their chemical characterization indicated siderophores of twelve fungi to be hydroxamates while one isolate Emericella nidulans exhibited a novel behaviour. Emericella nidulans a producer of both; hydroxamate and catecholate siderophore, a behaviour uncommon amongst fungi. The data revealed that exploring manglicolous fungi could reveal novelty in structural diversity of siderophore reflecting their unique ecological roles like avoiding siderophore piracy and oxidative stress for reclamation of mangrove associated coastal habitats.
\end{abstract}

Key words - chemical characterization - fungi - mangrove

\section{Introduction}

Iron is the macro molecule required for normal processes in microorganisms but its insoluble form present in earth's surface makes it unavailable for microbes. According to Holinsworth \& Martin (2009), marine environment has high nitrate low chlorophyll region (HNLC) in which iron is also in extremely low condition and affects the productivity of primary producers. This was supported by Gledhill \& Buck (2012). They stated that in marine environment, cycles of trace metals in oceans has become a matter of concern. Fe has received major importance amongst all other metals in oceans as its concentration controls primary productivity. Strategy followed by organisms in iron stressing condition is to produce low molecular weight compound called as siderophores. Siderophore forms complex with Fe (III) and makes iron available for phytoplankton in case of surface waters (Barbeau et al. 2001, Hunter \& Boyd 2007).

Mangrove ecosystem is well known for its potential biodiversity (Sahoo \& Dhal 2009). Gilna and Khaleel (2011) revealed that mangrove ecosystem is an ideal environment for fungal diversity. However, siderophore of this ecosystem is not well studied (Trivedi et al. 2016). There are various factors which affect the diversity of manglicolous fungi in mangrove plant viz. age of the mangrove, diversity of mangrove plant species and the physico-chemical features of mangrove habitat including temperature, salinity, and tidal range (Jones 2000). They include mostly marine fungi with a small group of terrestrial fungi. Marine fungi encountered on various parts of mangroves 



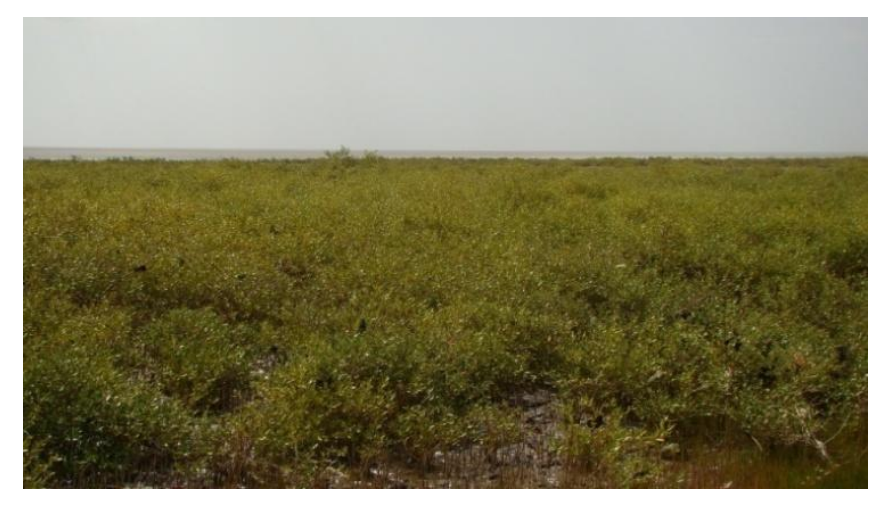

Fig. 1 Luxuriant growth of Avicennia marina at Ghogha

(Kohlmeyer and Kohlmeyer 1979). Fungi occurring in mangrove environment can also be categorized as saprophytic, pathogenic, endophytic, phosphate solubilizing and cellulose degrading fungi on basis of their different ecological roles. Saprophytic fungi are fundamental to many aspects of decomposition and energy flow in mangrove forests (Newell 1996).

In this study, manglicolous fungi (mangrove associated fungi) were examined for their ability to produce siderophores. Chemical characterization of siderophores was undertaken.

\section{Materials and Methods}

Four different mangrove samples as rhizosphere, rhizoplane, sediments and mangrove swamp water were collected from mangrove covered area at Ghogha Coast, Gujarat (Fig.1). These samples were treated in the laboratory. For analyses, $1 \mathrm{~g}$ of each, sediment and rhizosphere samples were mixed in $100 \mathrm{ml}$ sterile distilled water and shaken for $20 \mathrm{~min}$ to get their suspension. Similarly, roots with tightly adhering soil particles were cut into small pieces (approximately $1.5 \mathrm{~cm}$ ) and $1 \mathrm{~g}$ of these root pieces were vigorously mixed in sterile $100 \mathrm{ml}$ distilled water and shaken for $20 \mathrm{~min}$ to get the rhizoplane suspension. Each sample was serially diluted up to $10^{-3}$ and used for the isolation of manglicolous fungi. $100 \mathrm{uL}$ from above dilutions were then plated on various media viz. Potato Dextrose Agar (PDA), Sabouraud Agar (SBA), Czapeck Dox Agar (CDA) and Rose-Bengal Agar (RBA). Each medium was prepared in different concentrations sea water (0-100\%). and incubated at $30^{\circ} \mathrm{C}$ for 5-7 days. After proper incubation period, morphologically distinct fungal isolates were purified by repeated sub-culturing and stored on their respective media at $4{ }^{\circ} \mathrm{C}$ until future study. Morphological and microscopic observations were carried out for identification of the test fungi. Confirmed identification was carried out at the Agharker Research Institute, Pune.

All fungal isolates were subjected to screening for their ability to produce siderophores using various tests like $\mathrm{FeCl}_{3}$ test (Atkins et al. 1970), CAS assay test, CAS agar plate test (Schwayn \& Neilands 1987) and modified CAS agar plate test (Milagres et al. 1999). Throughout the study, all the glass wares were washed with $6 \mathrm{~N} \mathrm{HCl}$ to remove traces of iron. Grimm Allen medium (Grimm \& Allen 1954) used for the study was extracted with 8-hydroxyquinoline in chloroform to ensure complete removal of iron and $50 \mathrm{~mL}$ medium was dispensed in $250 \mathrm{~mL}$ flask. The flasks were inoculated with a disc of each thirteen test isolates and were placed on an environmental shaker (Thermo, MaxQ 400, USA) at $150 \mathrm{rpm}$ at $30^{\circ} \mathrm{C}$ for $7-9$ days. The media containing fungal growth were filtered using Whatman filter paper No.42 and the culture filtrates were subjected to various screening procedures as mentioned before.

The siderophores of manglicolous fungi were subjected to chemical characterization. Siderophores are chemically categorized according to the functional group used to co-ordinate with $\mathrm{Fe}^{3+}$, as hydroxamates, catecholates, carboxylates, mixed type siderophores and amphiphilic siderophores (produced exclusively by marine bacteria). $\mathrm{FeCl}_{3}$ test (Nielands 1981) and tetrazolium salt test (Snow 1954) for hydroxamate type of siderophores; $\mathrm{FeCl}_{3}$ test (Nielands 1981) and Arnow's test(Arnow 1937) for catecholate type of siderophore and spectrophotometric analysis (Shenker et al. 1992) for carboxylate type of siderophores were carried out. 


\section{Results}

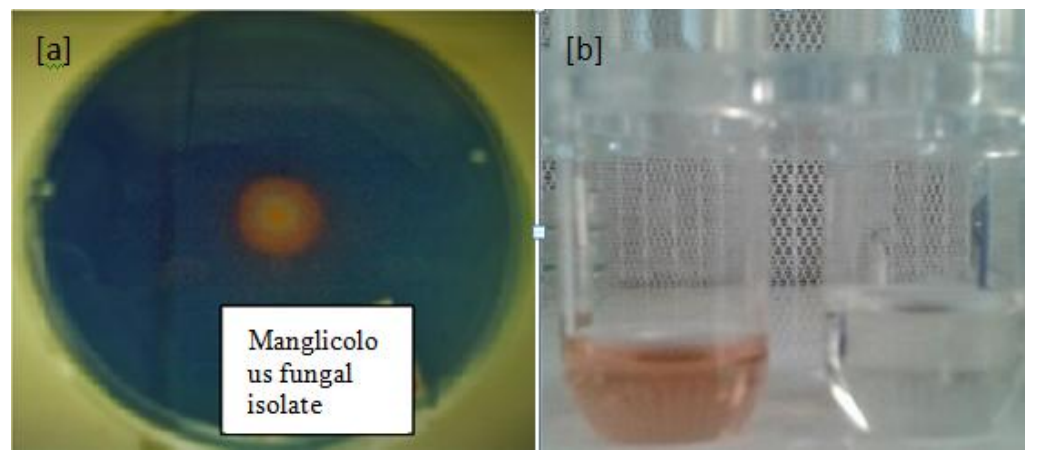

Fig. 2 Showing positive a. CAS agar test. b. Arnow's test by Emericella nidulans.

Thirteen manglicolous fungi were identified by using microscopic observations revealed that those fungi belong to Ascomycota; 10 belonged to Eurotiales and 3 belonged to family Pleosporales. Screening results revealed that all the thirteen test fungi exhibited siderophore production, indicating abundance of siderophores which solubilizes iron and makes it available for the other biodiversity present in mangrove ecosystem.

The chemical characterization shown that, all the test fungi gave positive tetrazolium salt test confirming hydroxamate nature of siderophore. None of the examined fungi produced carboxylates. One of the isolate, Emericella nidulans (confirmed identification carried out at Agharker Research Institute, Pune) gave positive Arnow's test as well (Fig.2), which is a novel behavior confirming catecholate nature of siderophore.

\section{Discussion}

Results of isolation were in agreement with report of Hyde and Jones (1988) which stated abundance of Ascomycota on marine and mangrove substrates. Behera et al. (2012) have also reported majority of marine fungi to belong to Ascomycota. Ravikumar \& Vittal (1996) reported the fungi colonizing different substrata of Rhizophora apiculata and $R$. mucronata from Pichavaram mangroves of Tamil Nadu, East coast of India and concluded that different substrata of the same host plant were colonized by different frequently occurring fungi. Borse et al. (2000) reported higher marine fungi in foam and intertidal wood and dead submerged wood of Avicennia marina from Daman coast. In this study 13 species of higher marine fungi (10 Ascomycetes, 3 Deuteromycetes) were recorded.

Screening results depicted the frequent occurrence of siderophore in manglicolous fungi might play an important role in the Fe cycle in mangrove ecosystem and thus help in increasing the primary productivity.

A novel behavior of E. Nidulans was observed during chemical characterization of siderophores. So far only one fungus Penicillium bilaii, isolated from marine environment has been reported to produce catecholate siderophore (Capon et al. 2007). Vala et al. (2000) had also reported Paecilomyces variotii; a marine derived fungal isolate producing carboxylate siderophore. Till then, carboxylate siderophores had been reported exclusively in Mucorales. The present study is the first report of catecholate siderophore from Emericella nidulans. Haas et al. (2003) reported that despite inherent production of three major siderophores fusigen, triacetylfusarinine $\mathrm{C}$, and ferri-crocin by Aspergillus nidulans, the fungus specifically utilizes catecholate-type siderophore Enterobactin. However, the present study reveals that manglicolous E. nidulans can produce catecholate siderophores as well which could be utilized by other non-catecholate siderophore producers. This study and earlier reports (Vala et al. 2000, Capon et al. 2007) suggest that exploration of marine habitats could probably reveal novelty in siderophore structures and functions.

Das et al. (2007) reported that simultaneous production of organic acid by most fungi could be probably restricting the occurrence of catecholates in fungi due to instability of ferric catecholates at 
acidic $\mathrm{pH}$. While lipophilicity, complex stability, high environmental $\mathrm{pH}$ and weak nitrogen metabolism are a few possible reasons for catecholate siderophores in bacteria, one of the possible reasons for occurrence of catecholate siderophores in E. nidulans could be the alkaline $\mathrm{pH}$, as the $\mathrm{pH}$ of mangrove ecosystem from which Emericella nidulans was isolated was noted to be 8.2.

A number of microorganisms have been reported to produce more than one siderophore with their synthesis under independent control. The reason behind siderophore diversity has not yet been completely understood. Recently, Lee et al. (2011) have suggested that diversity of siderophore is a consequence of interaction between siderophore producers (co-operators) and non-producers (cheaters). At times, it becomes beneficial to produce a siderophore unusable by the non-producers and avoid siderophore piracy. Adler et al. (2012) reported that besides their role in iron acquisition, siderophores may have other physiological roles as well. They have observed role of catecholate siderophores as protectors of oxidative stress.

Production of catecholate siderophore by Emericella nidulans could also be due to requirements as to avoid siderophore piracy and protection from oxidative stress. The present study confirms that marine environment can prove to be a good source of diversity of fungi with novel traits like siderophores with novel structures and functions. This is an encouraging observation for future research on protection and reclamation of mangrove ecosystem.

\section{Conclusion}

The present study indicated the exploitation of marine environment specifically mangrove habitat for isolation of manglicolous fungi and these fungal isolates then screened for siderophore production. Novel fungal isolate Emericella nidulans was found to produce hydroxamate as well as catecholate which were uncommon in fungi. So, marine environment again proved to be a source of novel secondary metabolites as well as fungal isolates.

\section{Acknowledgement}

AKV acknowledges the Department of Science and Technology, Government of India, New Delhi, for financial support under Women Scientist Scheme (WOS-A).

\section{References}

Adler C, Corbalan NS, Seyedsayamdost MR, Pomares, MF, de Cristo bal RE et al. 2012 - Catecholate siderophores protect bacteria from pyochelin toxicity. PLoS ONE 7(10), e46754.

Amin SA, Green DH, Al Waheeb D, Gärdes A, Carrano CJ. 2012 - Iron transport in the genus Marinobacter. Biometals 25, 135-147.

Arnow LE, 1937 - Colorimetric determination of compound of 3, 4-dihidroxyphenyl-alanine tyrosine mixtures. Journal of Biological Chemistry 118, 531-537.

Atkins CL, Neilands JB, Phaff HJ. 1970 - Rhodotorulic acid from species of Leucosporidium, Rhodospondium, Rhodotorula, Sporidiobolus and Sporobolomyces, and a new alanine containing ferrichrome from Cryptococcus meliviosum. Journal of Bacteriology 103, 722-733.

Barbeau K, Rue EL, Bruland KW, Butler A. 2001 - Photochemical cycling of iron in the surface ocean mediated by microbial iron (II)-binding ligands. Nature 413, 409-413.

Behera BC, Mishra RR, Thatoi HN. 2012 - Diversity of soil fungi from mangroves of Mahanadi delta, Orissa, India. Journal of Microbiolology Biotechnololgy Research 2, 375-378.

Borse BD, Kelkar DJ, Patil AC. 2000 - Frequency of occurrence of marine fungi from Pirotan Island (Gujarat), India. Geobios 27, 145-148.

Capon RJ, Stewart M, Ratnayake, R Lacey E, Gill JH. 2007 - Citromycetins and bilains A-C: New aromatic polyketides and diketopiperazines from Australian marine-derived and terrestrial Penicillium spp. Journal of Natural Products 70, 1746-1752.

Das A, Prasad R, Srivastava A, Giang PH, Bhatnagar K, Varma A. 2007 - Fungal Siderophores:Structure, Functions and Regulation In: Microbial Siderophores, Volume 12, Springer, Germany, pp 1-42. 
Gilna VV, Khaleel KM. 2011 - Diversity of fungi in mangrove ecosystem. Journal of Experimental Sciences 2(2), 47-48.

Gledhill M, Buck KN. 2012 - The organic complexation of iron in the marine environment: a review. Frontiers in Microbiology 3, 1-17.

Grimm PW, Allen PJ. 1954 - Promotion by zinc of the formation of cytochromes in Ustilaga sphaerogena. PI. Plant Physiology 29, 369.

Haas H, Schoeser M, Lesuisse E et al. 2003 - Chararacterization of the Aspergillus nidulans transporters for the siderophores enterobactin and triacetylfusarinine C. Biochemical Journal 371, 505-513.

Holinsworth B, Martin JD. 2009 - Siderophore production by marine derived fungi. Biometals 22(4), 625-632.

Hunter KA, Boyd PW. 2007 - Iron-binding ligands and their role in the ocean biogeochemistry of iron. Environmental Chemistry, 4, 221-232.

Hyde KD, Jones EBG. 1988 - Marine mangrove fungi. Marine Ecology 9, 15 - 33.

Jones EBG. 2000 - Marine fungi: some factors influencing biodiversity. Fungal Divers 4, 53-73

Kohlmeyer J, Kohlmeyer E. 1979 - Marine Mycology The Higher Fungi Academic Press New York.

Lee W, Minus Van Baalen, Vincent AA, Jansen. 2011 - An evolutionary mechanism for diversity in siderophore producing fungi. Ecology Letters 15(2), 119-125.

Milagres AMF, Napolea o D, Machuka A. 1999 - Detection of siderophore production from several fungi and bacteria by a modification of chrome azurol S (CAS) agar plate assay. Journal of Microbiological Methods 37, 1-7.

Newell SY. 1973 - Succession and role of fungi in the degradation of red mangrove seedlings. Estuarine microbial ecology 1, 467.

Nielands JB. 1981 - Methodology of siderophores. Structure and Bonding 58, 1-24.

Ravikumar DR, Vittal BPR. 1996 - Fungal diversity on decomposing biomass of mangrove plant Rhizophora in Pichavaram estuary east coast of India. Indian journal of marine sciences 25(2), $142-144$.

Sahoo K, Dhal NK. 2009 - Potential microbial diversity in mangrove ecosystem: A review. Indian Journal of Marine Sciences 38, 249-256.

Schwayn B, Neilands JB. 1987 - Universal chemical assay for the detection and determination of siderophores. Analytical Biochemistry 160, 47-56.

Shenker M, Olivar I, Helmann M, Hadar Y, Chen Y. 1992 - Utilization by tomatoes of iron mediated by a siderophore produced by Rhizopus arrhizus. Journal of plant Nutrition 15, 2173-2182.

Snow GA. 1954 - Mycobactin- A growth factor for Mycobacterium johnei II- Degradation and identification of fragments. Journal of Chemical Society 2588, 2596.

Trivedi HB, Vala AK, Dhrangadhariya J, Dave BP. 2016 - Marine-derived fungal siderophore: A perception Indian Journal of Geo Marine Sciences 45(3), 131-139.

Vala AK, Vaidya SY, Dube HC. 2000 - Siderophore production by facultative marine fungi. Indian Journal of Marine Science 29, 339-340. 